\title{
Analysis of COVID-19 Cases and Public Measures in China
}

\author{
Chun-Song $\mathrm{Hu}^{1}$ \\ Accepted: 21 July 2020 / Published online: 15 August 2020 \\ (C) Springer Nature Switzerland AG 2020
}

\begin{abstract}
This paper briefly analyzes COVID-19 cases during Wuhan lockdown and travel restrictions on 23 January 2020 to 23 June 2020, which included total confirmed, in critical condition, deaths, recovered, and suspected cases in China. Results showed that there were 28,942 suspected cases on February 8, 2020, at the peak; then, it almost declined continually to only several cases. Total confirmed cases were more than 80,000 on March 1, 2020, but less than 84,000, and deaths were more than 3000 on March 4, 2020, but less than 4640, totally, thanks for the right public measures for COVID-19 in China, such as the Wuhan City lockdown and travel restrictions for isolation; positive screening and testing; and establishing a Huoshenshan hospital, a Leishenshan hospital, and a number of Fangcang shelter hospitals, traditional Chinese medicine and a combination of Chinese and western medicine, and the launch of the clinical trials of antiviral drugs (Lianhua Qingwen, remdesivir, and chloroquine). In addition, the iRT-ABCDEF program is very useful to control domestic, imported, and asymptomatic cases. Cases in critical condition decrease continually after the peak of 11,977 cases on February 18,2020, and recovered cases increase continually to over 78,400 cases due to these right public measures and effective treatments. In recent months, there are only 2 deaths and only about ten cases in critical condition. All in all, these public measures in China are confirmed to be very effective and are worth conducting in countries worldwide.
\end{abstract}

Keywords COVID-19 $\cdot$ Infectious disease $\cdot$ Prevention $\cdot$ Public heath $\cdot$ SARS-CoV-2

\section{Introduction}

The outbreak of a severe respiratory disease $[1,2]$ at the end of 2019 has already been controlled successfully in China. This emerging infectious disease was ever named a novel coronavirus (2019-nCoV) pneumonia (NCP), and it is a major threat to public health according to the World Health Organization (WHO). Then, this coronavirus SARS-CoV-2 disease was named as COVID-19 by the WHO. Genome sequences from samples of patients confirmed that a novel RNA virus originated from its natural reservoir host—bats [3, 4]. But an animal just represents an intermediate host [5]. And pangolins should also be considered as possible hosts since metagenomic sequencing identified pangolin-associated coronaviruses [6]. The angiotensin-converting enzyme 2 (ACE2) is a possible entry

This article is part of the Topical Collection on Covid-19

Chun-Song $\mathrm{Hu}$

cnhucs@163.com

1 Department of Cardiovascular Medicine, Nanchang University, Hospital of Nanchang University, Jiangxi Academy of Medical Science, No. 461 Bayi Ave, Nanchang 330006, Jiangxi, China receptor for SARS-CoV-2 binding cell in humans. Here is a preliminary analysis of COVID-19 cases during Wuhan lockdown and travel restrictions on 23 January 2020 to 23 June 2020, which included total confirmed, in critical condition, deaths, recovered, and suspected cases in China.

\section{Methods}

\section{Data Collections}

Data were collected from January 23, 2020, the date of the Wuhan City lockdown and travel restrictions, to June 23, 2020. The information on total confirmed, in critical condition, deaths, recovered, and suspected cases was from the official website of the National Health Commission (http:// www.nhc.gov.cn/xcs/yqtb/list_gzbd.shtml), China.

\section{Statistical Analysis}

Statistical analysis was performed by SPSS 17 software with $t$ test for comparisons between two groups. $P$ values $<0.05$ were considered to denote statistical significance. 


\section{Results}

Data (Table 1 and Table 2) showed that there was 28,942 suspected cases on February 8, 2020, at the peak; then, it almost declined continually to only several cases. Total confirmed cases were more than 80,000 on March 1, 2020, but less than 84,000 , and deaths were more than 3000 on March 4 , 2020 , but less than 4640 , totally, thanks for the right public measures for COVID-19 in China, such as the Wuhan City lockdown and travel restrictions for isolation; positive screening and testing; and establishing a Huoshenshan hospital, a Leishenshan hospital, and a number of Fangcang shelter hospitals, traditional Chinese medicine (TCM) and a combination of Chinese and western medicine, and the launch of the clinical trials of antiviral drugs (Lianhua Qingwen, remdesivir, and chloroquine, etc.). Cases in critical condition decreased continually after the peak of 11,977 cases on February 18, 2020 , and recovered cases increased continually to over 78,400 due to these right public measures and effective treatments (Fig. 1). In recent months, there are only 2 deaths and only about ten cases in critical condition.

\section{Discussion}

So far, there were over 10 million confirmed cases in the globe after the pandemic of COVID-19 was declared by the WHO [7]. However, there were less 90,000 cases in China mainland. If compared with cases in major countries outside China, COVID-19 cases confirmed in China were earlier but less (definitely, $P<0.001$ ). This is highly linked to positive and effective public measures in China. With increasing understanding on the epidemiological, clinical, laboratory, and radiological characteristics; treatment; and clinical outcomes of COVID-19 patients $[8,9]$, there will be no possible second pandemic in China except for only some domestic, imported, and asymptomatic cases. Here is a brief summary on these powerful measures for prevention and control of COVID-19, and it will be helpful to fight against COVID-19 worldwide.

\section{Lockdown and Travel Restrictions for Isolation}

Since increasing evidence shows that human-to-human transmission has occurred among close contacts $[10,11]$, it is easy to understand that lockdown is very helpful to curb the spread of the epidemic. And, its effect of delaying and halting the outward spread of the SARS-CoV-2 was very positive [12], but it needs to combine with the control of transmission in the community [13]. Extending the statutory holiday and adopting a flexible working system to encourage residents to stay at home, cut off the transmission, and protect vulnerable individuals will effectively isolate the source of infection [14]. COVID-19 more likely affects older males with comorbidities and can result in severe and even fatal respiratory diseases [15], but there is currently no evidence of maternal-fetal SARS-CoV-2 transmission since no positive results were reported in testing on neonatal throat swabs and breast-milk samples [16-18].

\section{Early Detection and Prohibition of Gatherings}

Due to rapid transmissions, large-scale public health interventions need to be implement immediately in cities and rural regions with the pandemic [19]. All visitors were required to wear masks and to be detected by the temperature tests in the very beginning to prevent the spread of COVID-19 due to fever in $83 \%$ patients and cough in $82 \%$ patients [15]. And, local high-hazard regions had undergone door-to-door screenings for fighting against COVID-19. All confirmed cases and close contacts had been promptly quarantined and closed down for early detection of suspected cases. In many cities and communities, electronic proofs of access and health were required for all visitors to prevent imported COVID19. A series of bans were issued for prohibiting gathering. Universities and middle schools carried out online courses. Since there were possible asymptomatic cases of potentially COVID-19 infection [20-22], it is very important for isolation of 2 weeks for those closely contacted with confirmed cases.

\section{Releasing Information and Avoiding Panic}

The epidemic information was released in time by radio and television, internet, mobile phone, and WeChat. The diagnostic methods and treating programs were unclassified, and experts were invited to popularize scientific propaganda so as to stabilize the mood of residents and avoid possible panic by effective psychological channels. Open letters were widely posted to the community to publicize the harm of COVID19 as effective preventive measures. Especially, a healthy lifestyle which includes five core elements, "environment-sleepemotion-exercise-diet" intervention [E(e)SEEDi], also named the magic "polypill" [23], is highly encouraged because of its improvement of one's immunity (Table 3 ).

\section{Special Hospitals and Clinical Trials for Antiviral Treatment}

In order to treat all COVID-19 patients in time, a Huoshenshan hospital, a Leishenshan hospital, and a number of Fangcang shelter hospitals [24] were established in Wuhan which were rare miracles, and significant medical supplies were offered for urgent needs, for example, N95 masks, protective clothings, and ECMO, and many medical teams and famous experts were sent to Wuhan. And, remdesivir [25, 26], a possible effective 
Table 1 Data on COVID-19 cases in China during January 23 to June 23, 2020

\begin{tabular}{|c|c|c|c|c|c|}
\hline Time & $\begin{array}{l}\text { Total } \\
\text { confirmed cases }\end{array}$ & $\begin{array}{l}\text { Cases in } \\
\text { critical condition }\end{array}$ & Deaths & $\begin{array}{l}\text { Recovered } \\
\text { cases }\end{array}$ & $\begin{array}{l}\text { Suspected } \\
\text { cases }\end{array}$ \\
\hline 23-June-2020 & 83,430 & 12 & 4634 & 78,428 & 18 \\
\hline 23-Мау-2020 & 82,974 & 8 & 4634 & 78,261 & 9 \\
\hline 22-Мау-2020 & 82,971 & 9 & 4634 & 78,258 & 6 \\
\hline 21-Мay-2020 & 82,971 & 8 & 4634 & 78,255 & 7 \\
\hline 20-May-2020 & 82,967 & 8 & 4634 & 78,249 & 7 \\
\hline 19-Мау-2020 & 82,965 & 9 & 4634 & 78,244 & 7 \\
\hline 18-Мау-2020 & 82,960 & 10 & 4634 & 78,241 & 3 \\
\hline 17-May-2020 & 82,954 & 8 & 4634 & 78,238 & 4 \\
\hline 16-May-2020 & 82,947 & 10 & 4634 & 78,227 & 4 \\
\hline 15-Мау-2020 & 82,941 & 11 & 4633 & 78,219 & 3 \\
\hline 14-Мау-2020 & 82,933 & 11 & 4633 & 78,209 & 4 \\
\hline 13-May-2020 & 82,929 & 9 & 4633 & 78,195 & 4 \\
\hline 12-Маy-2020 & 82,926 & 10 & 4633 & 78,189 & 4 \\
\hline 11-Мау-2020 & 82,919 & 10 & 4633 & 78,171 & 3 \\
\hline 10-Мау-2020 & 82,918 & 9 & 4633 & 78,144 & 3 \\
\hline 09-Мау-2020 & 82,918 & 9 & 4633 & 78,144 & 3 \\
\hline 08-Маy-2020 & 82,901 & 13 & 4633 & 78,120 & 4 \\
\hline 07-May-2020 & 82,886 & 18 & 4633 & 77,993 & 6 \\
\hline 06-Мау-2020 & 82,885 & 23 & 4633 & 77,957 & 4 \\
\hline 05-May-2020 & 82,883 & 26 & 4633 & 77,911 & 5 \\
\hline 04-Мау-2020 & 82,881 & 29 & 4633 & 77,853 & 2 \\
\hline 03-May-2020 & 82,880 & 33 & 4633 & 77,766 & 3 \\
\hline 02-May-2020 & 82,877 & 34 & 4633 & 77,713 & 10 \\
\hline 01-Мау-2020 & 82,875 & 37 & 4633 & 77,685 & 11 \\
\hline 30-April-2020 & 82,874 & 38 & 4633 & 77,642 & 9 \\
\hline 29-April-2020 & 82,862 & 41 & 4633 & 77,610 & 10 \\
\hline 28-April-2020 & 82,858 & 50 & 4633 & 77,578 & 10 \\
\hline 27-April-2020 & 82,836 & 50 & 4633 & 77,555 & 9 \\
\hline 26-April-2020 & 82,830 & 52 & 4633 & 77,474 & 10 \\
\hline 25-April-2020 & 82,827 & 51 & 4632 & 77,394 & 12 \\
\hline 24-April-2020 & 82,816 & 49 & 4632 & 77,346 & 17 \\
\hline 23-April-2020 & 82,804 & 57 & 4632 & 77,257 & 20 \\
\hline 22-April-2020 & 82,798 & 63 & 4632 & 77,207 & 20 \\
\hline 21-April-2020 & 82,788 & 78 & 4632 & 77,151 & 35 \\
\hline 20-April-2020 & 82,758 & 82 & 4632 & 77,123 & 37 \\
\hline 19-April-2020 & 82,747 & 81 & 4632 & 77,084 & 43 \\
\hline 18-April-2020 & 82,735 & 85 & 4632 & 77,062 & 48 \\
\hline 17-April-2020 & 82,719 & 85 & 4632 & 77,029 & 63 \\
\hline 16-April-2020 & 82,367 & 89 & 3342 & 77,944 & 62 \\
\hline 15-April-2020 & 82,341 & 95 & 3342 & 77,892 & 63 \\
\hline 14-April-2020 & 82,295 & 113 & 3342 & 77,816 & 73 \\
\hline 13-April-2020 & 82,249 & 116 & 3341 & 77,738 & 72 \\
\hline 12-April-2020 & 82,160 & 121 & 3341 & 77,663 & 72 \\
\hline 11-April-2020 & 82,052 & 139 & 3339 & 77,575 & 82 \\
\hline 10-April-2020 & 81,953 & 141 & 3339 & 77,525 & 44 \\
\hline 09-April-2020 & 81,907 & 144 & 3336 & 77,455 & 53 \\
\hline 08-April-2020 & 81,865 & 176 & 3335 & 77,370 & 73 \\
\hline 07-April-2020 & 81,802 & 189 & 3333 & 77,279 & 83 \\
\hline 06-April-2020 & 81,740 & 211 & 3331 & 77,167 & 89 \\
\hline 05-April-2020 & 81,708 & 265 & 3331 & 77,078 & 88 \\
\hline 04-April-2020 & 81,669 & 295 & 3329 & 76,964 & 107 \\
\hline 03-April-2020 & 81,639 & 331 & 3326 & 76,751 & 114 \\
\hline 02-April-2020 & 81,620 & 379 & 3322 & 76,571 & 135 \\
\hline 01-April-2020 & 81,589 & 429 & 3318 & 76,408 & 153 \\
\hline 31-March-2020 & 81,554 & 466 & 3312 & 76,238 & 172 \\
\hline 30-March-2020 & 81,518 & 528 & 3305 & 76,052 & 183 \\
\hline 29-March-2020 & 81,470 & 633 & 3304 & 75,770 & 168 \\
\hline 28-March-2020 & 81,439 & 742 & 3300 & 75,448 & 174 \\
\hline 27-March-2020 & 81,394 & 921 & 3295 & 74,971 & 184 \\
\hline 26-March-2020 & 81,340 & 1034 & 3292 & 74,588 & 189 \\
\hline 25-March-2020 & 81,285 & 1235 & 3287 & 74,051 & 159 \\
\hline 24-March-2020 & 81,218 & 1399 & 3281 & 73,650 & 134 \\
\hline 23-March-2020 & 81,171 & 1573 & 3277 & 73,159 & 132 \\
\hline 22-March-2020 & 81,093 & 1749 & 3270 & 72,703 & 136 \\
\hline 21-March-2020 & 81,054 & 1845 & 3261 & 72,244 & 118 \\
\hline 20-March-2020 & 81,008 & 1963 & 3255 & 71,740 & 106 \\
\hline 19-March-2020 & 80,967 & 2136 & 3248 & 71,150 & 104 \\
\hline 18-March-2020 & 80,928 & 2314 & 3245 & 70,420 & 105 \\
\hline 17-March-2020 & 80,894 & 2622 & 3237 & 69,601 & 119 \\
\hline
\end{tabular}


Table 1 (continued)

\begin{tabular}{|c|c|c|c|c|c|}
\hline Time & $\begin{array}{l}\text { Total } \\
\text { confirmed cases }\end{array}$ & $\begin{array}{l}\text { Cases in } \\
\text { critical condition }\end{array}$ & Deaths & $\begin{array}{l}\text { Recovered } \\
\text { cases }\end{array}$ & $\begin{array}{l}\text { Suspected } \\
\text { cases }\end{array}$ \\
\hline 16-March-2020 & 80,881 & 2830 & 3226 & 68,679 & 128 \\
\hline 15-March-2020 & 80,860 & 3032 & 3213 & 67,749 & 134 \\
\hline 14-March-2020 & 80,844 & 3226 & 3199 & 66,911 & 113 \\
\hline 13-March-2020 & 80,824 & 3610 & 3189 & 65,541 & 115 \\
\hline 12-March-2020 & 80,813 & 4020 & 3176 & 64,111 & 147 \\
\hline 11-March-2020 & 80,793 & 4257 & 3169 & 62,793 & 253 \\
\hline 10-March-2020 & 80,778 & 4492 & 3158 & 61,475 & 285 \\
\hline 09-March-2020 & 80,754 & 4794 & 3136 & 59,897 & 349 \\
\hline 08 -March-2020 & 80,735 & 5111 & 3119 & 58,600 & 421 \\
\hline 07-March-2020 & 80,695 & 5264 & 3097 & 57,065 & 458 \\
\hline 06-March-2020 & 80,651 & 5489 & 3070 & 55,404 & 502 \\
\hline 05-March-2020 & 80,552 & 5737 & 3042 & 53,726 & 482 \\
\hline 04-March-2020 & 80,409 & 5952 & 3012 & 52,045 & 522 \\
\hline 03-March-2020 & 80,270 & 6416 & 2981 & 49,856 & 520 \\
\hline 02-March-2020 & 80,151 & 6806 & 2943 & 47,204 & 587 \\
\hline 01-March-2020 & 80,026 & 7110 & 2912 & 44,462 & 715 \\
\hline 29-February-2020 & 79,824 & 7365 & 2870 & 41,625 & 851 \\
\hline 28-February-2020 & 79,251 & 7664 & 2835 & 39,002 & 1418 \\
\hline 27-February-2020 & 78,824 & 7952 & 2788 & 36,117 & 2308 \\
\hline 26-February-2020 & 78,497 & 8346 & 2744 & 32,495 & 2358 \\
\hline 25-February-2020 & 78,064 & 8752 & 2715 & 29,745 & 2491 \\
\hline 24-February-2020 & 77,658 & 9126 & 2663 & 27,323 & 2824 \\
\hline 23-February-2020 & 77,150 & 9915 & 2592 & 24,734 & 3434 \\
\hline 22-February-2020 & 76,936 & 10,968 & 2442 & 22,888 & 4148 \\
\hline 21-February-2020 & 76,288 & 11,477 & 2345 & 20,659 & 5365 \\
\hline 20-February-2020 & 75,465 & 11,633 & 2236 & 18,264 & 5206 \\
\hline 19-February-2020 & 74,576 & 11,864 & 2118 & 16,155 & 4922 \\
\hline 18-February-2020 & 74,185 & 11,977 & 2004 & 14,376 & 5248 \\
\hline 17-February-2020 & 72,436 & 11,741 & 1868 & 12,552 & 6242 \\
\hline 16-February-2020 & 70,548 & 10,644 & 1770 & 10,844 & 7264 \\
\hline 15-February-2020 & 68,500 & 11,272 & 1665 & 9419 & 8228 \\
\hline 14-February-2020 & 66,492 & 11,053 & 1523 & 8096 & 8969 \\
\hline 13-February-2020 & 63,851 & 10,204 & 1380 & 6723 & 10,109 \\
\hline 12-February-2020 & 59,804 & 8030 & 1367 & 5911 & 13,435 \\
\hline 11-February-2020 & 44,653 & 8204 & 1113 & 4740 & 16,067 \\
\hline 10-February-2020 & 42,638 & 7333 & 1016 & 3996 & 21,675 \\
\hline 09-February-2020 & 40,171 & 6484 & 908 & 3281 & 23,589 \\
\hline 08-February-2020 & 37,198 & 6188 & 811 & 2649 & 28,942 \\
\hline 07-February-2020 & 34,546 & 6101 & 722 & 2050 & 27,657 \\
\hline 06-February-2020 & 31,161 & 4821 & 636 & 1540 & 26,359 \\
\hline 05-February-2020 & 28,018 & $3859^{*}$ & 563 & 1153 & 24,702 \\
\hline 04-February-2020 & 24,324 & 3219 & 490 & 892 & 23,260 \\
\hline 03-February-2020 & 20,438 & 2788 & 425 & 632 & 23,214 \\
\hline 02-February-2020 & 17,205 & 2296 & 361 & 475 & 21,558 \\
\hline 01 -February-2020 & 14,380 & 2110 & 304 & 328 & 19,544 \\
\hline 31-January-2020 & 11,791 & 1795 & 259 & 243 & 17,988 \\
\hline 30-January-2020 & 9692 & 1527 & 213 & 171 & 15,238 \\
\hline 29-January-2020 & 7711 & 1370 & 170 & 124 & 12,167 \\
\hline 28-January-2020 & 5974 & 1239 & 132 & 103 & 9239 \\
\hline 27-January-2020 & 4515 & 976 & 106 & 60 & 6973 \\
\hline 26-January-2020 & 2744 & 461 & 80 & 51 & 5794 \\
\hline 25-January-2020 & 1975 & 324 & 56 & 49 & 2684 \\
\hline 24-January-2020 & 1287 & 237 & 41 & 38 & 1965 \\
\hline 23-January-2020 & 830 & 177 & 25 & 34 & 1072 \\
\hline
\end{tabular}

Table 2 Analysis of CIVID-19 cases during January 23 to June 23, 2020 in China

\begin{tabular}{lccrrrr}
\hline Cases ltimes & $20,200,123$ & $20,200,223$ & $20,200,323$ & $20,200,423$ & $20,200,523$ & $20,200,623$ \\
\hline Total confirmed & 830 & $77150^{*}$ & $81171^{*}$ & $82804^{*}$ & $82974^{*}$ & $83430^{*}$ \\
In critical condition & 177 & $9915^{*}$ & $1573^{*}$ & $57^{*}$ & $8^{*}$ & $12^{*}$ \\
Deaths & 25 & $2592^{*}$ & $3277^{*}$ & $4632^{*}$ & $4634^{*}$ & $4634^{*}$ \\
Recovered & 34 & $24734^{*}$ & $73159^{*}$ & $77257^{*}$ & $78261^{*}$ & $78428^{*}$ \\
Suspected & 1072 & $3434^{*}$ & $132^{*}$ & $20^{*}$ & $9 *$ & $18^{*}$ \\
\hline
\end{tabular}

$* P<0.001$ when compared with cases on January 23, 2020 


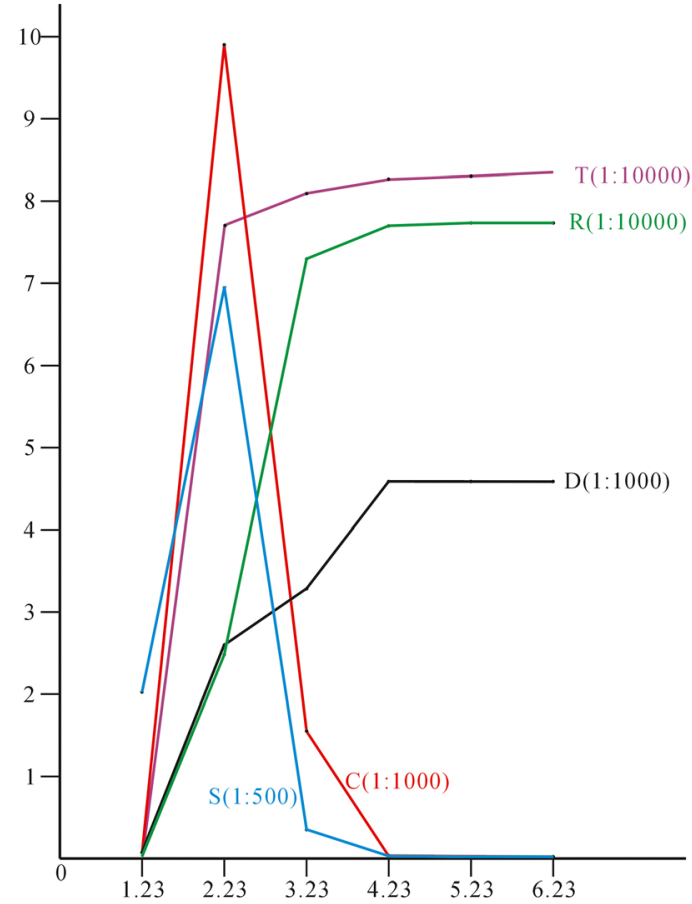

Fig. 1 The curve on analysis of COVID-19 cases in China during January 23 to June 23,2020 . T(1:10000): total confirmed cases; R(1:10000): recovered cases; $\mathrm{D}(1: 1000)$ : deaths; $\mathrm{C}(1: 1000)$ : cases in critical condition; $\mathrm{S}(1: 500)$ : suspected cases antiviral drug for the treatment of COVID-19, was imported. Of course, its clinical effect needs to be confirmed by further and large-scale clinical trials.

With the in-depth studies on clinical characteristics of COVID-19 [27]; the establishment of rapid diagnostic methods; integration of traditional Chinese and western medicine; screening specific antiviral drugs in the labs; the launch of the clinical trials on Lianhua Qingwen, remdesivir, and chloroquine due to effective inhibition of SARS-CoV-2 in vitro and in vivo [28, 29], and other drugs; and rapid developing of COVID-19 vaccines, as well as the developed iRT-ABCDEF program [30-34] for domestic, imported, and asymptomatic cases, rolling victory had been achieved in China. In addition, convalescent plasma therapy was well tolerated and could potentially improve the clinical outcomes in severe COVID-19 cases [35].

\section{Conclusion and Prospects}

Powerful public measures for combating COVID-19 were in time, rational, right, and scientific. These measures were confirmed to be very effective and are worth conducting worldwide including Brazil and Italy [36] and were highly praised by the WHO and many countries. It is time for global cooperation and information sharing [37, 38]. People should pay more attention to the role of laws on public health since Health in All Laws is a better strategy for global health. With the

Table 3 "Environment-sleep-emotion-exercise-diet" intervention [E(e)SEEDi] and immunity

\begin{tabular}{|c|c|c|}
\hline E(e)SEEDi & Immunity (-) & Immunity (+) \\
\hline External environment & $\begin{array}{l}\text { Air pollution and noise or e-noise } \\
\text { Lack of social relationship and friendship } \\
\text { Lower social-economic status and income }\end{array}$ & $\begin{array}{l}\text { Enjoy sunshine } \\
\text { Often communicate with your loved ones }\end{array}$ \\
\hline Internal (oneself) environment & $\begin{array}{l}\text { Chronic or acute infection } \\
\text { Other chronic diseases, such as cardiovascular } \\
\text { disease, type } 2 \text { diabetes, and cancer }\end{array}$ & $\begin{array}{l}\text { Wash your hands often } \\
\text { Wearing a mask }\end{array}$ \\
\hline Sleep & $\begin{array}{l}\text { Insomnia or sleep not enough } \\
\text { Obstructive sleep apnea (OSA) } \\
\text { Stay up late }\end{array}$ & $\begin{array}{l}\text { Get enough sleep } \\
(7-9 \text { h/night }) \\
\text { Have a nap at noon }\end{array}$ \\
\hline Emotion & $\begin{array}{l}\text { Anxiety or depression } \\
\text { Pessimism and thinking the worst } \\
\text { Have work pressure or overload } \\
\text { Death of a spouse }\end{array}$ & $\begin{array}{l}\text { Smile, be humorous, and optimistic } \\
\text { Daydreaming, having a good mood } \\
\text { Laughter (stress hormone levels drop) } \\
\text { or limit pressure }\end{array}$ \\
\hline Exercise & $\begin{array}{l}\text { Lack of physical activity } \\
\text { Rarely walk or often drive }\end{array}$ & $\begin{array}{l}\text { Adhere to massage } \\
\text { Keep exercising } \\
\text { Maintain a healthy weight } \\
\text { Ride a bike } \\
\text { Take deep breaths and practice yoga }\end{array}$ \\
\hline Diet & $\begin{array}{l}\text { Excessive drinking } \\
\text { Overuse of antibiotics } \\
\text { Smoking }\end{array}$ & $\begin{array}{l}\text { Do not smoke and limit alcohol consumption } \\
\text { Drink more water including honey or lemon water } \\
\text { Eat lots of fruits and vegetables } \\
\text { Morning or afternoon tea (black tea, green tea, ginseng tea) } \\
\text { Often eat deep sea fish, chicken soup, and yogurt }\end{array}$ \\
\hline
\end{tabular}


further studies on structures of the SARS-CoV-2 [39-41], and a SI(R) model on the COVID-19 pandemic [42], effective vaccines have been developed and now clinical trials are underway for better fighting against COVID-19.

Acknowledgments The reviewers and editors are gratefully acknowledged for their critical review. Thanks to Xueyan Shan, English teacher of Nanchang University (Master of English translation major from Sichuan International Studies University), for English revision and language polishing.

Author Contribution $\mathrm{CH}$ designed the study, performed the statistical analysis, and contributed to the writing of the paper.

Data Availability All data are available online.

\section{Compliance with Ethical Standards}

Conflict of Interest The author declares that he has no conflict of interest.

Ethics Approval Obtained from Nanchang University, Hospital of Nanchang University, Jiangxi Academy of Medical Science, China.

\section{References}

1. Zhu N, Zhang D, Wang W, Li X, Yang B, Song J, et al. A novel coronavirus from patients with pneumonia in China, 2019. N Engl J Med. 2020;382(8):727-33.

2. Ren LL, Wang YM, Wu ZQ, Xiang ZC, Guo L, Xu T, et al. Identification of a novel coronavirus causing severe pneumonia in human: a descriptive study. Chin Med J. 2020;133(9):1015-24.

3. Zhou P, Yang XL, Wang XG, Hu B, Zhang L, Zhang W, et al. A pneumonia outbreak associated with a new coronavirus of probable bat origin. Nature. 2020;579(7798):270-3.

4. Wu F, Zhao S, Yu B, Chen YM, Wang W, Song ZG, et al. A new coronavirus associated with human respiratory disease in China. Nature. 2020;579(7798):265-9.

5. Lu R, Zhao X, Li J, Niu P, Yang B, Wu H, et al. Genomic characterisation and epidemiology of 2019 novel coronavirus: implications for virus origins and receptor binding. Lancet. 2020;395(10224):565-74.

6. Lam TTY, Shum MHH, Zhu HC, Tong YG, Ni XB, Liao YS, et al. Identifying SARS-CoV-2 related coronaviruses in Malayan pangolins. Nature. 2020; Mar 26. https://doi.org/10.1038/s41586-0202169-0.

7. The World Health Organization (WHO). Coronavirus disease (COVID-2019) situation reports. 2020; Available from: https:// www.who.int/emergencies/diseases/novel-coronavirus-2019/ situation-reports/

8. Huang C, Wang Y, Li X, Ren L, Zhao J, Hu Y, et al. Clinical features of patients infected with 2019 novel coronavirus in Wuhan, China. Lancet. 2020;395(10223):497-506.

9. Wang D, Hu B, Hu C, Zhu F, Liu X, Zhang J, et al. Clinical characteristics of 138 hospitalized patients with 2019 novel coronavirus-infected pneumonia in Wuhan, China. JAMA. 2020;323(11):1061-9.

10. Li Q, Guan X, Wu P, Wang X, Zhou L, Tong Y, et al. Early transmission dynamics in Wuhan, China, of novel coronavirusinfected pneumonia. N Engl J Med. 2020;382(13):1199-207.
11. Chan JF, Yuan S, Kok KH, To KK, Chu H, Yang J, et al. A familial cluster of pneumonia associated with the 2019 novel coronavirus indicating person-to-person transmission: a study of a family cluster. Lancet. 2020;395(10223):514-23.

12. Tian H, Liu Y, Li Y, Wu CH, Chen B, Kraemer MUG, et al. An investigation of transmission control measures during the first 50 days of the COVID-19 epidemic in China. Science. 2020;368(6491):638-42.

13. Chinazzi M, Davis JT, Ajelli M, Gioannini C, Litvinova M, Merler S, et al. The effect of travel restrictions on the spread of the 2019 novel coronavirus (COVID-19) outbreak. Science. 2020;368(6489):395-400.

14. Phan LT, Nguyen TV, Luong QC, Nguyen TV, Nguyen HT, Le $\mathrm{HQ}$, et al. Importation and human-to-human transmission of a novel coronavirus in Vietnam. N Engl J Med. 2020;382(9):872-4.

15. Chen N, Zhou M, Dong X, Qu J, Gong F, Han Y, et al. Epidemiological and clinical characteristics of 99 cases of 2019 novel coronavirus pneumonia in Wuhan, China: a descriptive study. Lancet. 2020;395(10223):507-13.

16. Chen H, Guo J, Wang C, Luo F, Yu X, Zhang W, et al. Clinical characteristics and intrauterine vertical transmission potential of COVID-19 infection in nine pregnant women: a retrospective review of medical records. Lancet. 2020;395(10226):809-15.

17. Stower H. Lack of maternal-fetal SARS-CoV-2 transmission. Nat Med. 2020;26(3):312.

18. Chen L, Li Q, Zheng D, Jiang H, Wei Y, Zou L, et al. Clinical characteristics of pregnant women with Covid-19 in Wuhan, China. N Engl J Med. 2020;382(25):e100.

19. Wu JT, Leung K, Leung GM. Nowcasting and forecasting the potential domestic and international spread of the 2019-nCoV outbreak originating in Wuhan, China: a modelling study. Lancet. 2020;395(10225):689-97.

20. Liu YC, Liao CH, Chang CF, Chou CC, Lin YR. A locally transmitted case of SARS-CoV-2 infection in Taiwan. N Engl J Med. 2020;382(11):1070-2.

21. Rothe C, Schunk M, Sothmann P, Bretzel G, Froeschl G, Wallrauch C, et al. Transmission of 2019-nCoV infection from an asymptomatic contact in Germany. N Engl J Med. 2020;382(10):970-1.

22. Hoehl S, Rabenau H, Berger A, Kortenbusch M, Cinatl J, Bojkova $\mathrm{D}$, et al. Evidence of SARS-CoV-2 infection in returning travelers from Wuhan, China. N Engl J Med. 2020;382(13):1278-80.

23. $\mathrm{Hu} \mathrm{C}$. Grants supporting research in China. Eur Heart J. 2018;39(25):2342-4.

24. Chen S, Zhang Z, Yang J, Wang J, Zhai X, Bärnighausen T, et al. Fangcang shelter hospitals: a novel concept for responding to public health emergencies. Lancet. 2020;395(10232):1305-14.

25. Holshue ML, DeBolt C, Lindquist S, Lofy KH, Wiesman J, Bruce $\mathrm{H}$, et al. First case of 2019 novel coronavirus in the United States. N Engl J Med. 2020;382(10):929-36.

26. Grein J, Ohmagari N, Shin D, Diaz G, Asperges E, Castagna A, et al. Compassionate use of remdesivir for patients with severe Covid-19. N Engl J Med. 2020;382(24):2327-36.

27. Guan WJ, Ni ZY, Hu Y, Liang WH, Ou CQ, He JX, et al. Clinical characteristics of coronavirus disease 2019 in China. N Engl J Med. 2020;382(18): 1708-20.

28. Runfeng L, Yunlong H, Jicheng H, Weiqi P, Qinhai M, Yongxia S, et al. Lianhua Qingwen exerts anti-viral and anti-inflammatory activity against novel coronavirus (SARS-CoV-2). Pharmacol Res. 2020;156:104761.

29. Wang M, Cao R, Zhang L, Yang X, Liu J, Xu M, et al. Remdesivir and chloroquine effectively inhibit the recently emerged novel coronavirus (2019-nCoV) in vitro. Cell Res. 2020;30(3):269-71.

30. Hu CS, Tkebuchava T. SARS and its treatment strategies. Asian Pac J Trop Med. 2019;12(3):95-7. 
31. Hu CS, Wu QH, Hu DY, Tkebuchava T. Treatment of chronic heart failure in the 21st century: a new era of biomedical engineering has come. Chronic Dis Transl Med. 2018;5(2):75-88.

32. Hu CS. Intervention of RT-ABCDEF for cancer. Croat Med J. 2019;60(1):55-7.

33. Hu C, Tkebuchava T, Hu D. Managing acute myocardial infarction in China. Eur Heart J. 2019;40(15):1179-81.

34. Hu CS. A comprehensive strategy for managing arrhythmogenic right ventricular cardiomyopathy. Turk Kardiyol Dern Ars. 2020;48(2):88-95.

35. Duan K, Liu B, Li C, Zhang H, Yu T, Qu J, et al. Effectiveness of convalescent plasma therapy in severe COVID-19 patients. Proc Natl Acad Sci U S A. 2020;117(17):9490-6.

36. de Souza Ferreira LP, Valente TM, Tiraboschi FA, da Silva GP. Description of Covid-19 cases in Brazil and Italy. SN Comprehen Clin Med. 2020;2:497-500.

37. Coronavirus: three things all governments and their science advisers must do now. Nature. 2020;579(7799):319-20.
38. Moorthy V, Henao Restrepo AM, Preziosi MP, Swaminathan S. Data sharing for novel coronavirus (COVID-19). Bull World Health Organ. 2020;98(3):150.

39. Wrapp D, Wang N, Corbett KS, Goldsmith JA, Hsieh CL, Abiona $\mathrm{O}$, et al. Cryo-EM structure of the 2019-nCoV spike in the prefusion conformation. Science. 2020;367(6483):1260-3.

40. Walls AC, Park YJ, Tortorici MA, Wall A, McGuire AT, Veesler D. Structure, function and antigenicity of the SARS-CoV-2 spike glycoprotein. Cell. 2020;181(2):281-92 e6.

41. Yan R, Zhang Y, Li Y, Xia L, Guo Y, Zhou Q. Structural basis for the recognition of the SARS-CoV-2 by full-length human ACE2. Science. 2020;367(6485):1444-8.

42. Rocchi E, Peluso S, Sisti D, Carletti M. A possible scenario for the Covid-19 epidemic, based on the SI(R) model. SN Comprehen Clin Med. 2020;2:501-3.

Publisher's Note Springer Nature remains neutral with regard to jurisdictional claims in published maps and institutional affiliations. 